This is an electronic reprint of the original article. This reprint may differ from the original in pagination and typographic detail.

Author(s): Pirinen, Pekka; Srivastava, P. C.; Suhonen, Jouni; Kortelainen, Markus

Title: $\quad$ Shell-model study on event rates of lightest supersymmetric particles scattering off $83 \mathrm{Kr}$ and $125 \mathrm{Te}$

Year: $\quad 2016$

Version:

Please cite the original version:

Pirinen, P., Srivastava, P. C., Suhonen, J., \& Kortelainen, M. (2016). Shell-model study on event rates of lightest supersymmetric particles scattering off $83 \mathrm{Kr}$ and $125 \mathrm{Te}$.

Physical Review D, 93(9), Article 095012.

https://doi.org/10.1103/PhysRevD.93.095012

All material supplied via JYX is protected by copyright and other intellectual property rights, and duplication or sale of all or part of any of the repository collections is not permitted, except that material may be duplicated by you for your research use or educational purposes in electronic or print form. You must obtain permission for any other use. Electronic or print copies may not be offered, whether for sale or otherwise to anyone who is not an authorised user. 


\title{
Shell-model study on event rates of lightest supersymmetric particles scattering off ${ }^{83} \mathrm{Kr}$ and ${ }^{125} \mathrm{Te}$
}

\author{
P. Pirinen, ${ }^{1}$ P. C. Srivastava, ${ }^{2}$ J. Suhonen, ${ }^{1}$ and M. Kortelainen ${ }^{1,3}$ \\ ${ }^{1}$ University of Jyvaskyla, Department of Physics, P.O. Box 35 (YFL), FI-40014 University of Jyvaskyla, Finland \\ ${ }^{2}$ Department of Physics, Indian Institute of Technology, Roorkee 247667, India \\ ${ }^{3}$ Helsinki Institute of Physics, University of Helsinki, P.O. Box 64, FI-00014 University of Helsinki, Finland
}

(Received 4 December 2015; published 17 May 2016)

\begin{abstract}
We investigate the elastic and inelastic scattering of lightest supersymmetric particle (LSP) dark matter off two possible target nuclei, ${ }^{83} \mathrm{Kr}$ and ${ }^{125} \mathrm{Te}$. For the nuclear-structure calculations, we employ the nuclear shell model using recently generated realistic interactions. We have condensed the nuclear-physics contribution to a set of nuclear-structure factors that are independent of the adopted supersymmetric (SUSY) model. Total event rates are then easily calculated by combining the nuclear-structure factors with SUSY parameters of choice. In particular, ${ }^{125} \mathrm{Te}$ shows promise as a detector material with both the elastic and inelastic channels yielding an appreciable nuclear response.
\end{abstract}

DOI: 10.1103/PhysRevD.93.095012

\section{INTRODUCTION}

Since the initial idea [1], dark matter has been actively investigated by the scientific community. Even today, little is certain of the nature of this elusive constituent of our Universe. In recent cosmic microwave background (CMB) experiments [2,3], it is evident that normal luminous matter makes up only a small fraction of all the matter in the Universe, and most of the matter in our Universe is dark. Most of this dark matter is likely to be cold, i.e., nonrelativistic at the time of freeze-out. Two of the usual candidates for the cold dark matter (CDM) component are massive compact halo objects (MACHOs), such as brown dwarfs or neutron stars, and weakly interacting massive particles (WIMPs). Only a fraction of the CDM component can consist of baryonic MACHOs [4,5], which gives rise to interest in nonbaryonic WIMPs.

Theoretical motivation for WIMPs can be found in several models, such as Kaluza-Klein models with universal extra dimensions [6,7], technicolor [8,9], and little Higgs models with $T$ parity $[10,11]$. We choose to work with supersymmetry (SUSY) [12], which offers a compelling and natural WIMP candidate, as the lightest supersymmetric particle (LSP), the lightest neutralino, is supposedly charge neutral and (nearly) stable, and, furthermore, has a suitable relic density to constitute a large portion of CDM [13]. As dark matter itself, supersymmetry is a proposition which has not directly been experimentally detected, but offers a theoretical explanation to a number of observed phenomena. Thus, direct detection of dark matter could also be a step towards validating supersymmetry.

Direct detection experiments are set in deep mine laboratories under extensive shielding to eliminate background radiation as effectively as possible. The aim in most of these experiments is to detect a nuclear recoil signal in a scattering with a dark matter particle, in some detectors combined with the simultaneous detection of a possible deexcitation gamma ray associated with inelastic scattering. The mechanisms of a WIMP-nucleus interaction are not known, so an ideal detector nucleus would be one that is sensitive to both coherent and incoherent interactions, and also allows inelastic scattering of WIMPs. Therefore, many popular detector nuclei have an odd nucleon and a low first excited state to which a transition might be allowed in a WIMP-nucleus scattering.

There are currently numerous experimental groups dedicated to direct detection of dark matter. At present, the most favored nuclear targets include ${ }^{19} \mathrm{~F}$ (SIMPLE [14], PICASSO [15]), ${ }^{73} \mathrm{Ge}$ (CDMS [16], EDELWEISS [17]) ${ }^{127} \mathrm{I}$ (KIMS [18], DAMA/LIBRA [19]), ${ }^{129,131}$ Xe (XENON [20], XMASS [21], LZ [22]), and ${ }^{133} \mathrm{Cs}$ (KIMS). Aside from the above nuclei already used in detectors, there exist some other theoretically viable candidates [23], such as ${ }^{83} \mathrm{Kr}$ and ${ }^{125} \mathrm{Te}$, which to our knowledge have not been examined thoroughly within a complete microscopic nuclear framework. Both of these nuclei have properties which make them very interesting as detector materials. ${ }^{83} \mathrm{Kr}$ has a remarkably low first excited state at only $9.4 \mathrm{keV}$, which might open new possibilities for inelastic scattering of WIMPs. The first excited state of ${ }^{125} \mathrm{Te}$ is quite low as well, at roughly $35 \mathrm{keV}$. Moreover, the transitions to the excited states are GamowTeller like, with $\Delta J=1$. On the negative side, both of these isotopes have a relatively low natural abundance. Still, the theoretical interest for these nuclei is significant and experimental feasibility is to be investigated.

Nuclear physics and nuclear structure play an important role in dark matter detectors [24]. Being able to accurately calculate the wave functions for the low-lying states of the target nucleus is a crucial aspect in calculating the event rate for WIMP-nucleus scattering. In early calculations $[23,25,26]$, the nuclear contribution was usually evaluated 
by rudimentary methods. In Ref. [27], the interacting bosonfermion model (IBFM) was used to examine spin-dependent WIMP-nucleus scattering. A shell-model study of nuclear physics aspects in LSP-nucleus scattering was made in Ref. [28] for ${ }^{23} \mathrm{Na},{ }^{71} \mathrm{Ga},{ }^{73} \mathrm{Ge}$, and ${ }^{127} \mathrm{I}$, and the results were compared to an earlier MQPM (microscopic quasiparticle-phonon model) calculation of [29]. Another comprehensive shell-model calculation was made along the lines of Refs. [28,29] in Ref. [30] for the popular detector nuclei ${ }^{127} \mathrm{I},{ }^{129} \mathrm{Xe},{ }^{131} \mathrm{Xe},{ }^{133} \mathrm{Cs}$. An older shell-model study [31] includes also ${ }^{125} \mathrm{Te}$, but calculations therein include only spin-dependent elastic scattering. In recent work [32,33], the odd xenons were studied for the elastic and inelastic channels of the WIMP-nucleus scattering by using recently developed shell-model interactions. Reference [34] extends this study to even- $A$ xenon isotopes. In Ref. [35], the methods of Ref. [32] were used to examine spin-dependent WIMP scattering off a wider variety of nuclei. In two very recent articles [36,37], an examination was made for ${ }^{83} \mathrm{Kr}$ and ${ }^{125} \mathrm{Te}$, using the same $\mathrm{SM}$ calculations as the present work, but the focus there was more on model independent event rates rather than nuclear structure.

Contrary to a constant rate, one would expect to see an annual modulation in the WIMP-nucleus scattering event rate [38]. The annual modulation is a result of the Earth's orbital motion around the Sun. As the Sun moves through the Galactic dark matter halo, there is effectively a constant wind of dark matter particles through the Solar system. The Earth then orbits against the wind for half of its period, encountering relatively more WIMPs than on the other half of the period, when the Earth's velocity is more parallel to the wind. Although the modulation effect is typically predicted to be quite small, it might be a valuable signal for direct WIMP detection. The DAMA group has observed a modulation signal to a confidence level of $9.3 \sigma$ [39] after 14 years of data taking, and recently a similar modulation has been observed by the CoGeNT Collaboration [40]. Whether the observed modulation is due to dark matter remains to be seen, as undisputed evidence is still lacking. The annual modulation is especially important if the WIMP is not very heavy, i.e., has a mass of the order of $10 \mathrm{GeV}$ or less [41].

In this work, we use the nuclear shell model (SM) to calculate the nuclear structure of ${ }^{83} \mathrm{Kr}$ and ${ }^{125} \mathrm{Te}$. We then use the generated wave functions to calculate the nuclear structure-dependent parts of the total event rates of elastic and inelastic LSP-nucleus scattering. The nuclear responses of our target nuclei are compared to each other and to results on other targets obtained in other similar works. Total event rates are trivially obtainable from the nuclearstructure parts for any choice of SUSY parameters.

\section{SHELL-MODEL STRUCTURE OF THE TARGET NUCLEI}

For ${ }^{83} \mathrm{Kr}$, shell-model calculations have been carried out in the 28-50 valence shell composed of the $1 p_{3 / 2}, 0 f_{5 / 2}$,
$1 p_{1 / 2}, 0 g_{9 / 2}$ orbitals. The calculations have been performed with a recently available effective interaction, $\mathrm{jj} 44 \mathrm{~b}$, by Brown and Lisetskiy [42], and shell-model code NuShellX [43]. The single-particle energies employed in conjunction with the jj44b interaction are $-9.6566,-9.2859,-8.2695$, and $-5.8944 \mathrm{MeV}$ for the $1 p_{3 / 2}, 0 f_{5 / 2}, 1 p_{1 / 2}, 0 g_{9 / 2}$ orbitals, respectively. The positive-parity spectrum is well reproduced by the present calculation although some negative-parity states are predicted very low in energy. The first excited $7 / 2^{+}$state is predicted at $100 \mathrm{keV}$ with respect to the $9 / 2^{+}$state, while the corresponding experimental value is $9 \mathrm{keV}$. For ${ }^{83} \mathrm{Kr}$, we performed a fullfledged calculation with no truncations.

Shell-model calculations for ${ }^{125} \mathrm{Te}$ were carried out in the 50-82 valence shell composed of the orbits $0 g_{7 / 2}, 1 d_{5 / 2}$, $0 h_{11 / 2}, 2 s_{1 / 2}$, and $1 d_{3 / 2}$ with the SN100PN interaction due to Brown et al. $[44,45]$. The shell-model code NuShellX was again used to perform the calculation. This interaction has four parts: neutron-neutron, neutron-proton, protonproton and Coulomb repulsion between the protons. The single-particle energies for the neutrons are -10.6089 , $-10.289,-8.017,-8.694$, and $-8.816 \mathrm{MeV}$ for the $0 g_{7 / 2}, 1 d_{5 / 2}, 1 d_{3 / 2}, 2 s_{1 / 2}$, and $0 h_{11 / 2}$ orbitals, respectively. Those for the protons are $0.807,1.562,3.316,3.224$, and $3.605 \mathrm{MeV}$. In the present calculation, we slightly modified the single-particle energy of the $\nu 1 d_{3 / 2}$ orbital from -8.717 to $-8.017 \mathrm{MeV}$ (changing by $700 \mathrm{keV}$ ). We performed the shell-model calculation using a truncation because of the large matrix dimension involved in the present calculation. Therefore, we allowed the two valence protons to occupy only the $\pi 0 g_{7 / 2}$ and $\pi 1 d_{5 / 2}$ orbits, and for neutrons we completely filled the $\nu 0 g_{7 / 2}$ and $\nu 1 d_{5 / 2}$ orbits, and put a minimum of six neutrons in the $\nu 0 h_{11 / 2}$ orbit. For ${ }^{125} \mathrm{Te}$, the ground state was not reproduced by the calculation, but the order of the important positive-parity states is correct.

The computed low-lying energy spectra for ${ }^{83} \mathrm{Kr}$ (labeled full) and ${ }^{125} \mathrm{Te}$ (labeled Truncation 1) are given in Figs. 1 and 2, respectively, and compared with experimental data. In the figures, results are given also for different truncation schemes, described later. In the case of ${ }^{83} \mathrm{Kr}$, the order of the positive-parity states is very nicely reproduced, although the calculated energy difference between the $9 / 2^{+}$and $7 / 2^{+}$states is larger than the experimental one. Then again, the first negative-parity states, $1 / 2^{-}$ and $5 / 2^{-}$, are predicted very low by our shell-model calculation. For ${ }^{125} \mathrm{Te}$, the negative-parity states are also systematically predicted to much lower energies than their experimental counterparts. Therefore, an $11 / 2^{-}$ground state is predicted, while the experimental ground state is $1 / 2^{+}$. The first $1 / 2^{+}$state is predicted at $18 \mathrm{keV}$. The positive parity spectrum is in decent agreement with the experimental data. One has to also keep in mind that all of the interesting states in the presently discussed nuclei are within an energy range of $150 \mathrm{keV}$ and perfect prediction of 


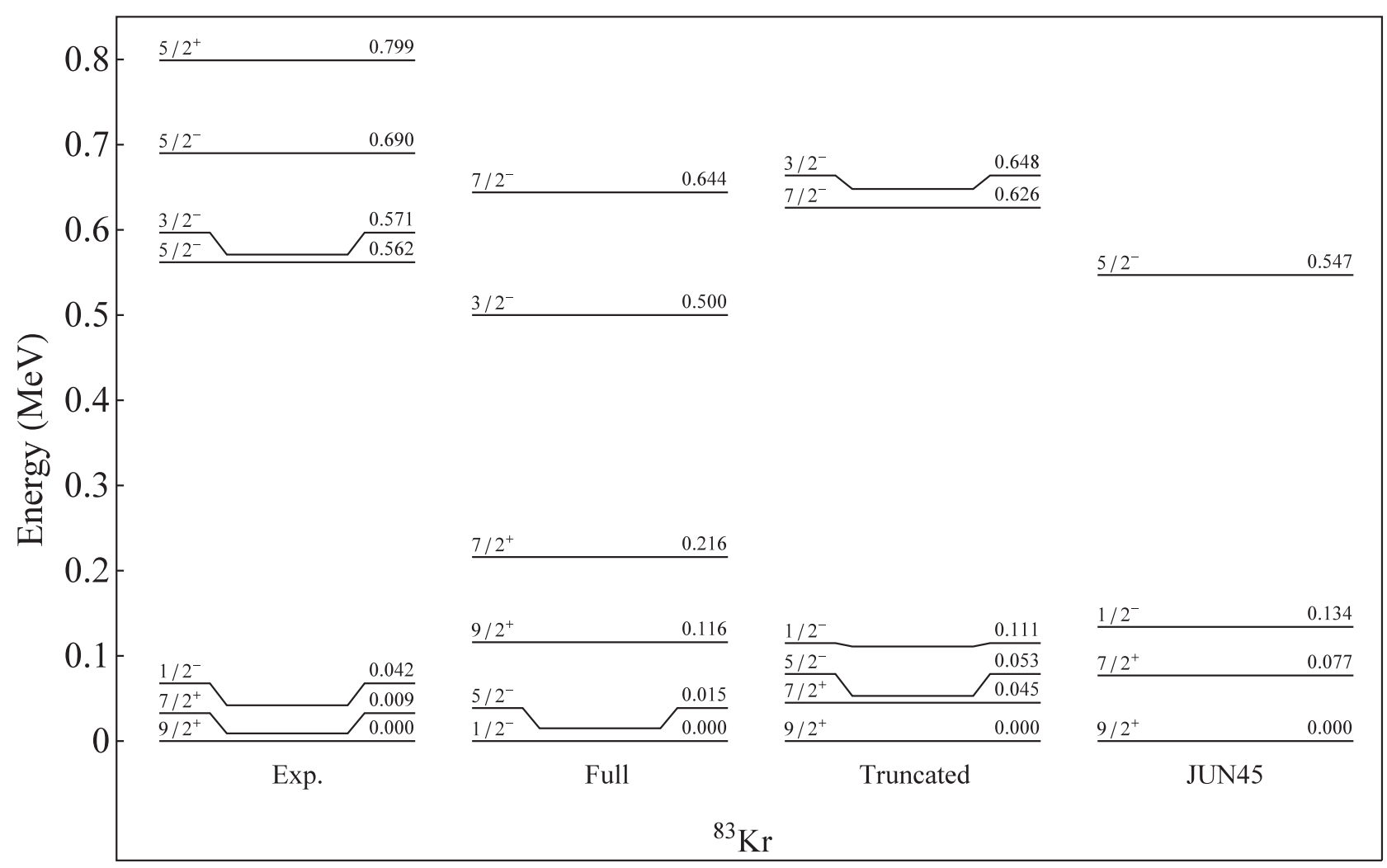

FIG. 1. Experimental and computed energy levels of ${ }^{83} \mathrm{Kr}$. Levels are shown for full and truncated calculations with the jj44b interaction and a full calculation with the JUN45 interaction (see text).

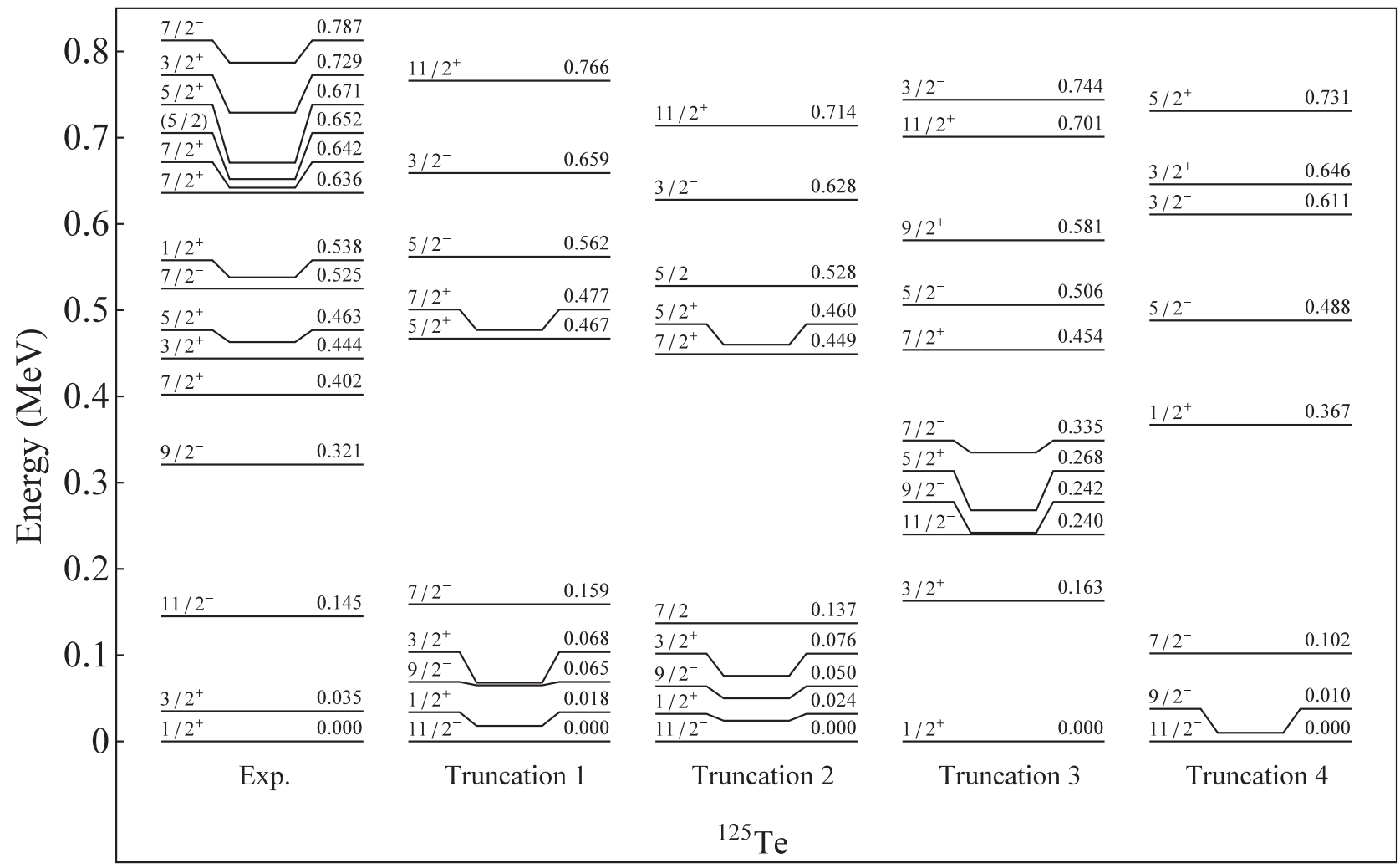

FIG. 2. Experimental and computed energy levels of ${ }^{125}$ Te. Levels are shown for four different truncations (see text) with the SN100PN interaction. 
TABLE I. Experimental and calculated $B(\mathrm{E} 2)$ and $B(\mathrm{M} 1)$ values for ${ }^{83} \mathrm{Kr}\left(7 / 2_{1}^{+} \rightarrow 9 / 2_{1}^{+}\right)$and ${ }^{125} \mathrm{Te}\left(3 / 2_{1}^{+} \rightarrow 1 / 2_{1}^{+}\right)$with standard effective charges: $\mathrm{e}_{\text {eff }}^{\pi}=1.5 e, \mathrm{e}_{\text {eff }}^{\nu}=1.0 e$ for ${ }^{125} \mathrm{Te}$ and $\mathrm{e}_{\mathrm{eff}}^{\pi}=1.5 e, \mathrm{e}_{\mathrm{eff}}^{\nu}=0.5 e$ for ${ }^{83} \mathrm{Kr}$. Bare $g$ factors $g_{s}^{\text {eff }}=g_{s}^{\text {free }}$ were used. The experimental $\gamma$-ray energies corresponding to these transitions are also shown.

\begin{tabular}{lcccccc}
\hline \hline & & \multicolumn{2}{c}{$B($ E2 $)($ W.u. $)$} & & \multicolumn{2}{c}{$B(\mathrm{M} 1)($ W.u. $)$} \\
\cline { 3 - 4 } \cline { 7 - 7 } Nucleus & $E_{\gamma}(\mathrm{keV})$ & Exp. & SM & & Exp. & \multicolumn{1}{c}{ SM } \\
\hline${ }^{83} \mathrm{Kr}$ & 9.4 & $21.0(10)$ & 11.62 & & $0.00933(4)$ & 0.0028 \\
${ }^{125} \mathrm{Te}$ & 35.5 & $11.9(24)$ & 6.03 & & $0.0226(4)$ & 0.00564 \\
\hline \hline
\end{tabular}

their energies constitutes a considerable challenge for nuclear-structure calculations.

In Table I, we have compared the calculated and experimental $B(\mathrm{E} 2)$ and $B(\mathrm{M} 1)$ values using standard effective charges and bare gyromagnetic factors. The overall agreement is reasonable; i.e., the theoretical transition matrix elements are expected to deviate from the experimental ones by less than a factor of 2 . The calculated values of electric quadrupole and magnetic dipole moments are shown in Table II. These results are also in reasonable agreement with available experimental data although the magnetic moments are predicted systematically larger than the experimental values. The present calculation reproduces correctly the sign of the quadrupole and magnetic moments. It is possible to further improve the results for $B(\mathrm{M} 1)$ and magnetic moments by adjusting the values of the effective $g$ factors. We have chosen to work with the bare $g$ factors because of the ambiguity related to determining well-justified effective ones.

Leading configurations for the two lowest-lying states are given in Table III. The structure of the $9 / 2^{+}$state of ${ }^{83} \mathrm{Kr}$ is $\nu\left(g_{9 / 2}^{-3}\right)$ (with probability $\sim 16 \%$ ). The same configuration is predicted for the first excited $7 / 2^{+}$state $(\sim 26 \%)$. For ${ }^{125} \mathrm{Te}$, the configurations of the $1 / 2_{1}^{+}$and $3 / 2_{1}^{+}$states are $\nu 2 s_{1 / 2}$ and $\nu 1 d_{5 / 2}$, respectively.

The experimental energy spectra of ${ }^{125} \mathrm{Te}$ and ${ }^{83} \mathrm{Kr}$, as shown above, are not exactly reproduced by our shellmodel calculation. As a measure of accuracy we have shown the experimentally observed electromagnetic properties compared to computed ones in Tables I and II. To

TABLE II. Electric quadrupole moments $Q_{s}$ (in $e$ b), and magnetic moments $\mu$ (in $\mu_{N}$ ). The effective charges $e_{p}=1.5 e$, $e_{n}=0.5 e$, and $g_{s}^{\text {eff }}=g_{s}^{\text {free }}$ were used in the calculation. Experimental values were taken from Ref. [46].

\begin{tabular}{lccccr}
\hline \hline \multirow{2}{*}{ Nucleus } & $J^{\pi}$ & $Q_{s}^{\operatorname{Exp}}$ & $Q_{s}^{\text {SM }}$ & $\mu_{\operatorname{Exp}}$ & $\mu_{\mathrm{SM}}$ \\
\hline${ }^{83} \mathrm{Kr}$ & $9 / 2^{+}$ & $0.26(3)$ & 0.34 & $-0.970669(3)$ & -1.412 \\
& $7 / 2^{+}$ & $0.495(10)$ & 0.41 & $-0.943(2)$ & -1.099 \\
${ }^{125} \mathrm{Te}$ & $1 / 2^{+}$ & N/A & N/A & $-0.8885051(4)$ & -1.598 \\
& $3 / 2^{+}$ & $-0.31(2)$ & -0.18 & $0.605(4)$ & 0.950 \\
\hline \hline
\end{tabular}

TABLE III. The most important configurations of the lowestlying positive-parity states of ${ }^{83} \mathrm{Kr}$ and ${ }^{125} \mathrm{Te}$.

\begin{tabular}{lcc}
\hline \hline Nucleus & $J^{\pi}$ & Configuration \\
\hline${ }^{83} \mathrm{Kr}$ & $9 / 2^{+}$ & $\nu g_{9 / 2}^{-3}(16 \%)$ \\
& $7 / 2^{+}$ & $\nu g_{9 / 2}^{-3}(26 \%)$ \\
${ }^{125} \mathrm{Te}$ & $1 / 2^{+}$ & $\nu s_{1 / 2}(68 \%)$ \\
& $3 / 2^{+}$ & $\nu d_{3 / 2}(53 \%)$ \\
\hline \hline
\end{tabular}

gain insight to the inaccuracy resulting from the interaction and truncations, we performed additional calculations with more severe truncations for both of our target nuclei. For ${ }^{83} \mathrm{Kr}$, we also performed a full calculation with a different interaction, JUN45 [47]. For ${ }^{125} \mathrm{Te}$, we only had one suitable interaction available, SN100PN.

We truncate the model space in the ${ }^{83} \mathrm{Kr}$ case for both protons and neutrons as follows: a minimum of 4 particles on the $0 f_{5 / 2}$ orbital, a minimum of 2 particles on the $1 p_{3 / 2}$ orbital, and no restrictions on the $1 p_{1 / 2}$ and $0 g_{9 / 2}$ orbitals. The resulting energy spectra for the full and truncated calculations for ${ }^{83} \mathrm{Kr}$ are presented in Fig. 1 along with the JUN45 result and the experimental spectrum. The truncated spectrum is curiously more reminescent of the experimental spectrum than the full calculation. The JUN45 interaction results in the best correspondence with experiment, but the $B(\mathrm{M} 1)$ value is almost vanishing for this interaction as shown in Table IV. The $B(\mathrm{M} 1)$ value of the truncated calculation is also further away from the experimental value than that of the full calculation. We believe the full jj44b calculation to be the most accurate description of the structure of the low-lying positive-parity states in ${ }^{83} \mathrm{Kr}$.

The four different truncations for the ${ }^{125} \mathrm{Te}$ case are described in Table V, and shall be referred to as truncations 1-4. Truncation 1 is the least truncated valence space, which was already described before. The energy spectra of these truncations are given in Fig. 2. We notice that slightly altering the truncation does not change the order of the lowest-lying energy levels by comparing truncations 1 and 2. Truncation 3 produces the correct ground state,

TABLE IV. Magnetic moments $\mu$ (in $\mu_{N}$ ) and B(M1) values (in W.u.) of ${ }^{83} \mathrm{Kr}$ and ${ }^{125} \mathrm{Te}$ compared between different truncations.

\begin{tabular}{lclll}
\hline \hline Nucleus & Setup & \multicolumn{1}{c}{$\mu\left(1 / 2_{\mathrm{gs}}^{+}\right)$} & $\mu\left(3 / 2_{1}^{+}\right)$ & \multicolumn{1}{c}{$B(\mathrm{M} 1)$} \\
\hline${ }^{83} \mathrm{Kr}$ & Experimental & $-0.970669(3)$ & $-0.943(2)$ & $0.00933(4)$ \\
& Full jj44b & -1.412 & -1.099 & 0.0028 \\
& Truncated jj44b & -1.517 & -1.203 & 0.0018 \\
& Full JUN45 & -1.457 & -1.185 & 0.0000 \\
${ }^{125} \mathrm{Te}$ & Experimental & $-0.8885051(4)$ & $0.605(4)$ & $0.0226(4)$ \\
& Truncation 1 & -1.598 & 0.950 & 0.00564 \\
& Truncation 2 & -1.588 & 0.957 & 0.00616 \\
& Truncation 3 & -1.718 & 1.009 & 0.00018 \\
& Truncation 4 & -1.900 & 0.888 & 0.00009 \\
\hline \hline
\end{tabular}


TABLE V. The truncations applied to the shell-model calculations on ${ }^{125} \mathrm{Te}$ for proton $(\pi)$ orbitals and neutron $(\nu)$ orbitals. The particle number restrictions set for each orbital are given in columns 2-5 for the four truncations used.

\begin{tabular}{lcccc}
\hline \hline & \multicolumn{4}{c}{ Truncation } \\
\cline { 2 - 5 } Orbital & 1 & 2 & 3 & 4 \\
\hline$\pi 0 g_{7 / 2}$ & free & free & free & free \\
$\pi 1 d_{5 / 2}$ & free & empty & empty & empty \\
$\pi 1 d_{3 / 2}$ & empty & empty & empty & empty \\
$\pi 2 s_{1 / 2}$ & empty & empty & empty & empty \\
$\pi 0 h_{11 / 2}$ & empty & empty & empty & empty \\
$\nu 0 g_{7 / 2}$ & full & full & full & full \\
$\nu 1 d_{5 / 2}$ & full & full & full & full \\
$\nu 1 d_{3 / 2}$ & free & free & min. 2 & full \\
$\nu 2 s_{1 / 2}$ & free & free & free & free \\
$\nu 0 h_{11 / 2}$ & min. 6 & min. 6 & min. 6 & free \\
\hline \hline
\end{tabular}

but there is a large energy gap to the first excited $3 / 2^{+}$state. Truncation 4 is clearly inaccurate due to the valence space being very restricted in that case. The magnetic properties of the different calculations show that truncations 1 and 2 are closest to the experimental values while the $B(\mathrm{M} 1)$ values given by truncations 3 and 4 are too small by a factor of over a 100 . Thus, in the calculations for the rest of the paper, we use the least truncated calculation, truncation 1 , for best reproducing the overall experimental properties.

We have performed shell-model calculations for ${ }^{125} \mathrm{Te}$ in a truncated 50-82 model space to make the shell-model calculations feasible. We observed that if we slightly change the truncation the energy levels are not shifting significantly. The $11 / 2^{-}$state at lower excitation energy is not due to the restricted calculation but to the effective interaction which needs further tuning. For the Te chain, even if we performed full-fledged calculations, e.g. in ${ }^{129,131} \mathrm{Te}$, the present interaction predicts an $11 / 2^{-}$state as the ground state. Previously the same set of truncation which we are using in the present work has been employed to explain experimentally observed $g$-factors for the lowlying states of ${ }^{125} \mathrm{Te}$ by the Canberra group, which is reported in Ref. [48].

\section{LSP-NUCLEUS SCATTERING EVENT RATES}

In this section, main ingredients entering the event rates of elastic and inelastic LSP-nucleus scatterings are discussed. The event rates are given in a form in which the parameters arising from supersymmetric theories and nuclear structure are separated as in Refs. [28,30]. This offers a chance to better discuss the nuclear physics aspects of WIMP detectors independently of any specific supersymmetric model. The expected annual modulation in the signal due to the Earth's motion around the Sun is also taken into account. In the following, the effect of the Earth's rotation around its axis has been omitted as negligible, and the orbits of the Earth around the Sun and the Sun around the Galaxy are taken to be circular with constant tangential speeds.

\section{A. Event rate of elastic scattering}

An expression for the total event rate of elastic LSPnucleus scattering was derived in Ref. [28],

$$
\begin{aligned}
\langle R\rangle= & {\left[\left(f_{\mathrm{A}}^{0}\right)^{2} D_{1}+2 f_{\mathrm{A}}^{0} f_{\mathrm{A}}^{1} D_{2}+\left(f_{\mathrm{A}}^{1}\right)^{2} D_{3}\right.} \\
& \left.+A^{2}\left(f_{\mathrm{S}}^{0}-f_{\mathrm{S}}^{1} \frac{A-2 Z}{A}\right)^{2} D_{4}\right] m_{\mathrm{det}}[\mathrm{kg}],
\end{aligned}
$$

where $m_{\mathrm{det}}$ is the mass of the detector in units of $\mathrm{kg}$, and $A$ and $Z$ are the nuclear mass number and proton number, respectively. Here the supersymmetry is confined in the scalar and axial-vector form factors $f_{\mathrm{S}}^{\rho}$ and $f_{\mathrm{A}}^{\rho}$, and the factors $D_{n}$ depend on the nuclear structure of the target nucleus and include the effect of annual modulation. The event rate is divided into a spin-dependent incoherent part, containing the first three terms, and a spin-independent coherent part, which is proportional to the square of the nuclear mass number $A$. The folding of the event rate with the LSP velocity distribution is also contained in the coefficients $D_{n}$.

The LSP velocity distribution is taken to be of a Maxwell-Boltzmann character and it can be written as

$$
f\left(\mathbf{v}, \mathbf{v}_{\mathrm{E}}\right)=\left(\sqrt{\pi} v_{0}\right)^{-3} e^{-\left(\mathbf{v}+\mathbf{v}_{\mathrm{E}}\right)^{2} / v_{0}^{2}},
$$

where $\mathbf{v}$ is the LSP velocity with respect to the earthbound detector. The velocity of the Earth with respect to the Galactic center can be written as

$$
\mathbf{v}_{\mathrm{E}}=\mathbf{v}_{0}+\mathbf{v}_{1}
$$

where $\mathbf{v}_{0}$ is the velocity of the Sun with respect to the galactic center and $\mathbf{v}_{1}$ is the velocity of the Earth (and thus the detector) relative to the Sun. To describe $\mathbf{v}_{1}$, the orbit of the Earth around the Sun is considered to be circular and the tangential speed constant. The angle between the ecliptic and the galactic equator is taken to be $60.2^{\circ}$. In a coordinate system, where $\hat{\mathbf{x}}_{2}$ points to the galactic north, $\hat{\mathbf{x}}_{3}$ is in the direction of the Sun's velocity, and $\hat{\mathbf{x}}_{1}=\hat{\mathbf{x}}_{2} \times \hat{\mathbf{x}}_{3}, \mathbf{v}_{1}$ can be expressed as

$\mathbf{v}_{1}=v_{1}\left[\sin (\alpha) \hat{\mathbf{x}}_{1}-\cos (\alpha) \cos (\gamma) \hat{\mathbf{x}}_{2}+\cos (\alpha) \sin (\gamma) \hat{\mathbf{x}}_{3}\right]$

where $v_{1}=30 \mathrm{~km} / \mathrm{s}$ and $\gamma$ is the angle between the normal of the ecliptic and the galactic plane $\left(\gamma \approx 29.8^{\circ}\right)$.

The nuclear-structure factors $D_{n}$ of Eq. (1) can be written as 


$$
\begin{array}{r}
D_{1}=\int_{-1}^{1} \int_{\psi_{\min }}^{\psi_{\max }} \int_{u_{\min }}^{u_{\max }} G(\psi, \xi) F_{00}(u) \Omega_{0}^{2} d \xi d \psi d u, \\
D_{2}=\int_{-1}^{1} \int_{\psi_{\min }}^{\psi_{\max }} \int_{u_{\min }}^{u_{\max }} G(\psi, \xi) F_{01}(u) \Omega_{0} \Omega_{1} d \xi d \psi d u, \\
D_{3}=\int_{-1}^{1} \int_{\psi_{\min }}^{\psi_{\max }} \int_{u_{\min }}^{u_{\max }} G(\psi, \xi) F_{11}(u) \Omega_{1}^{2} d \xi d \psi d u, \\
D_{4}=\int_{-1}^{1} \int_{\psi_{\min }}^{\psi_{\max }} \int_{u_{\min }}^{u_{\max }} G(\psi, \xi)|F(u)|^{2} d \xi d \psi d u,
\end{array}
$$

with the modulation function $G(\psi, \xi)$ defined as

$$
G(\psi, \xi)=\frac{\rho_{0}}{m_{\chi}} \frac{\sigma_{0}}{A m_{\mathrm{p}}}\left(\frac{1}{m_{\mathrm{p}} b}\right)^{2} \frac{e^{-\lambda^{2}}}{\sqrt{\pi}} \frac{c^{2}}{v_{0}} \psi e^{-\psi^{2}} e^{-2 \lambda \psi \xi},
$$

where $\rho_{0}=0.3 \mathrm{GeV} / \mathrm{cm}^{3}$ is the local WIMP density, $\sigma_{0} \approx 0.77 \times 10^{-38} \mathrm{~cm}^{2}, m_{\mathrm{p}}$ the mass of a proton, $m_{\chi}$ the LSP mass, and $b$ the harmonic oscillator length. In the above equations, $\psi=v / v_{0}, \lambda=v_{\mathrm{E}} / v_{0}$, and $u$ is the momentum transfer $q$ of the scattering process expressed in a dimensionless form (in natural units)

$$
u=q^{2} b^{2} / 2 .
$$

The factor $|F(u)|^{2}$ entering the coherent channel is the square of the nuclear form factor. The factors $F_{\rho \rho^{\prime}}(u)$ in Eqs. (5)-(7) are the nuclear spin structure functions (SSFs) defined as

$$
F_{\rho \rho^{\prime}}(u)=\sum_{\lambda, \kappa} \frac{\Omega_{\rho}^{(\lambda, \kappa)}(u) \Omega_{\rho^{\prime}}^{(\lambda, \kappa)}(u)}{\Omega_{\rho} \Omega_{\rho^{\prime}}},
$$

where

$$
\Omega_{\rho}=\Omega_{\rho}^{(0,1)}(0)
$$

are the static spin matrix elements (SSMEs), and

$$
\begin{aligned}
& \Omega_{\rho}^{(\lambda, \kappa)}(u) \\
& =\sqrt{\frac{4 \pi}{2 J_{i}+1}}\left(J_{f}\left\|\sum_{j=1}^{A}\left[\mathbf{Y}_{\lambda}\left(\Omega_{j}\right) \otimes \boldsymbol{\sigma}\right]_{\kappa} j_{\lambda}\left(\sqrt{u} r_{j}\right) \omega_{\rho}(j)\right\| J_{i}\right) .
\end{aligned}
$$

Above $\mathbf{Y}_{\lambda}$ is a spherical harmonic, $\Omega_{j}$ is a solid angle related to the position of the nucleon, $\boldsymbol{\sigma}$ is the Pauli operator, $j_{\lambda}$ is a spherical Bessel function, $\omega_{0}(j)=1$, and $\omega_{1}(j)=\tau_{3}(j)$ is the third component of isospin.

To find the limit $\psi_{\max }$, we write the speed of the Earth relative to the Sun as

$$
v_{\mathrm{E}}=\sqrt{v_{0}^{2}+v_{1}^{2}+2 v_{0} v_{1} \sin \gamma \cos \alpha},
$$

where $\alpha$ is the phase of the Earth on its orbit around the Sun ( $\alpha=0$ on June 2 and $\alpha=180^{\circ}$ on December 2). The velocity of the LSP with respect to the Galactic center is limited by the local Galactic escape velocity $v_{\text {esc }}=625 \mathrm{~km} / \mathrm{s}$, and using Eq. (3) we find that

$$
\left|\mathbf{v}+\mathbf{v}_{\mathrm{E}}\right|^{2}=v^{2}+v_{1}^{2}+v_{0}^{2}+2 \mathbf{v} \cdot \mathbf{v}_{\mathrm{E}}+2 \mathbf{v}_{0} \cdot \mathbf{v}_{1}<v_{\mathrm{esc}}^{2},
$$

which leads to an upper limit for the speed of the LSP. The lower limit for the LSP velocity is set by the detector threshold energy, which also sets a lower limit for the momentum transfer. The integration limits become

$$
\psi_{\min }=\frac{c}{v_{0}}\left(\frac{A m_{\mathrm{p}} Q_{\mathrm{thr}}}{2 \mu_{r}^{2}}\right)^{1 / 2},
$$

$$
\begin{gathered}
\psi_{\text {max }}=-\lambda \xi+\sqrt{\lambda^{2} \xi^{2}+\frac{v_{\text {esc }}^{2}}{v_{0}^{2}}-1-\frac{v_{1}^{2}}{v_{0}^{2}}-\frac{2 v_{0} v_{1}}{v_{0}^{2}} \sin \gamma \cos \alpha} \\
u_{\min }=A m_{\mathrm{p}} Q_{\mathrm{thr}} b^{2} \\
u_{\text {max }}=2\left(\psi \mu_{r} b v_{0} / c\right)^{2}
\end{gathered}
$$

where $Q_{\mathrm{thr}}$ is the detector threshold energy, and $\mu_{r}$ the reduced mass of the LSP-nucleus system.

Here it should be noted, that the values of $v_{\text {esc }}$ and $v_{0}$ are not uniform in the literature. Although the recommended standard value for $v_{0}$ is $220 \mathrm{~km} / \mathrm{s}$ [49], there is a report of model dependent estimates ranging from $(200 \pm 20)$ to $(279 \pm 33) \mathrm{km} / \mathrm{s}$ [50], and another study that predicts $(254 \pm 16) \mathrm{km} / \mathrm{s}$ [51]. The value of the local escape velocity in literature also varies by a lot. In our calculations, we have used the estimate $v_{\text {esc }}=625 \mathrm{~km} / \mathrm{s}$ of Ref. [38] as an upper limit. A recent result by the RAVE experiment, however, gives a best estimate of $v_{\text {esc }}=537_{-43}^{+59} \mathrm{~km} / \mathrm{s}$ [52]. The dependence of our results on the variance of $v_{\text {esc }}$ is very mild, but changing the value of $v_{0}$ can have a notable effect on the results.

\section{B. Event rate of inelastic scattering}

In the case of inelastic scattering, the form of the event rate is similar to Eq. (1) with the coherent $\left(\propto A^{2}\right)$ part omitted as only the incoherent part contributes. The inelastic event rate can thus be written as

$\langle R\rangle_{\text {inel }}=\left[\left(f_{\mathrm{A}}^{0}\right)^{2} E_{1}+2 f_{\mathrm{A}}^{0} f_{\mathrm{A}}^{1} E_{2}+\left(f_{\mathrm{A}}^{1}\right)^{2} E_{3}\right] m_{\text {det }}[\mathrm{kg}]$.

The nuclear structure is now confined in the three coefficients, 
$E_{1}=\int_{-1}^{1} \int_{\psi_{\min }}^{\psi_{\max }} \int_{u_{\min }}^{u_{\max }} G(\psi, \xi) F_{00}(u) \Omega_{0}^{2} d \xi d \psi d u$

$E_{2}=\int_{-1}^{1} \int_{\psi_{\min }}^{\psi_{\max }} \int_{u_{\min }}^{u_{\max }} G(\psi, \xi) F_{01}(u) \Omega_{0} \Omega_{1} d \xi d \psi d u$,

$E_{3}=\int_{-1}^{1} \int_{\psi_{\min }}^{\psi_{\max }} \int_{u_{\min }}^{u_{\max }} G(\psi, \xi) F_{11}(u) \Omega_{1}^{2} d \xi d \psi d u$,

where the modulation function, spin structure functions and static spin matrix elements are as defined in Sec. III A for elastic scattering. The integration limits, however, are different and can be expressed as [30]

$$
\begin{aligned}
& u_{\min }=\frac{1}{2} b^{2} \mu_{r}^{2} \frac{v_{0}^{2}}{c^{2}} \psi^{2}\left[1-\sqrt{1-\Gamma / \psi^{2}}\right]^{2}, \\
& u_{\max }=\frac{1}{2} b^{2} \mu_{r}^{2} \frac{v_{0}^{2}}{c^{2}} \psi^{2}\left[1+\sqrt{1-\Gamma / \psi^{2}}\right]^{2},
\end{aligned}
$$

where

$$
\Gamma=\frac{2 E^{*}}{\mu_{r} c^{2}} \frac{c^{2}}{v_{0}^{2}}
$$

with the nuclear excitation energy $E^{*}$. The LSP velocity, and thus $\psi$, is now limited from below by the nuclear excitation energy and from above by the Galactic escape velocity as before. The upper limit is thus the same as in Eq. (17), and the lower limit becomes simply

$$
\psi_{\min }=\sqrt{\Gamma} .
$$

\section{RESULTS AND DISCUSSION}

In this section, we present the results of our calculations along the lines presented in Sec. III. The results are given for the nuclear-structure parts of the event rates of both the elastic and inelastic channel of LSP-nucleus scattering.

\section{A. Results for elastic scattering}

A key element to a favorable scattering cross section are the static spin matrix elements of Eq. (12). In the elastic channel, we find appreciable values for both of our targets. The SSMEs are tabulated in Table VI for each of the truncation schemes presented in Sec. II. We notice that the effect of truncation (and interaction in the ${ }^{83} \mathrm{Kr}$ case) is quite small on the SSMEs for elastic scattering. A significant difference is found only in truncation 4 of ${ }^{125} \mathrm{Te}$, which is the most truncated case. The situation is different for inelastic scattering as will be shown in the next subsection. Aside from the large values of the SSMEs, a notable feature is the relative sign difference of the isoscalar and isovector SSME. The following calculations for structure factors are
TABLE VI. Static spin matrix elements in case of elastic LSPnucleus scattering off ${ }^{83} \mathrm{Kr}$ and ${ }^{125} \mathrm{Te}$.

\begin{tabular}{lccc}
\hline \hline Nucleus & Setup & $\Omega_{0}^{\text {el }}$ & $\Omega_{1}^{\text {el }}$ \\
\hline${ }^{83} \mathrm{Kr}$ & Full jj44b & 1.037 & -1.018 \\
& Truncated jj44b & 1.021 & -1.046 \\
& Full JUN45 & 1.086 & -1.016 \\
${ }^{125} \mathrm{Te}$ & Truncation 1 & 1.456 & -1.502 \\
& Truncation 2 & 1.442 & -1.495 \\
& Truncation 3 & 1.558 & -1.591 \\
& Truncation 4 & 1.721 & -1.733 \\
\hline \hline
\end{tabular}

made with our best estimate: the full jj44b calculation for ${ }^{83} \mathrm{Kr}$ and truncation 1 for ${ }^{125} \mathrm{Te}$.

The squared nuclear form factor and the spin structure functions of Eq. (11) for the elastic LSP-nucleus scattering off ${ }^{83} \mathrm{Kr}$ and ${ }^{125} \mathrm{Te}$ are given in Figs. 3(a) and 3(b), respectively, as a function of the dimensionless momentum transfer $u$. The overall quantitative behavior of the SSFs is similar for both of our target nuclei. With $u<1$, the SSFs follow quite closely the square of the nuclear form factor $F$, i.e., with a strong downward slope. In the case of ${ }^{125} \mathrm{Te}$, the

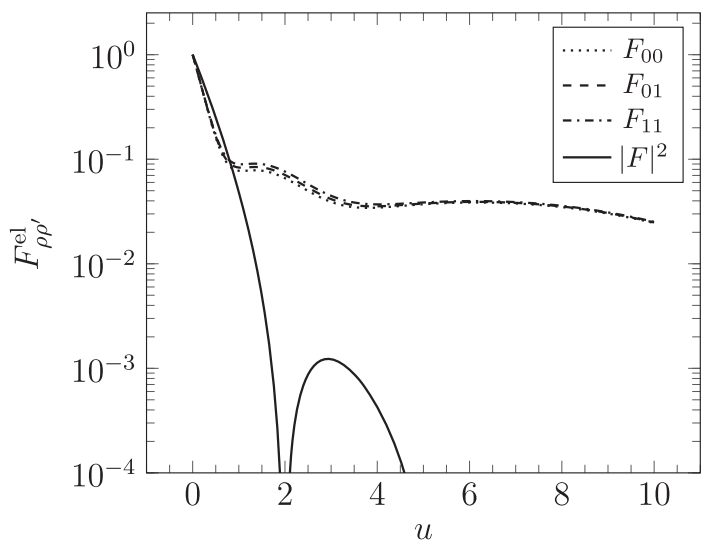

(a)

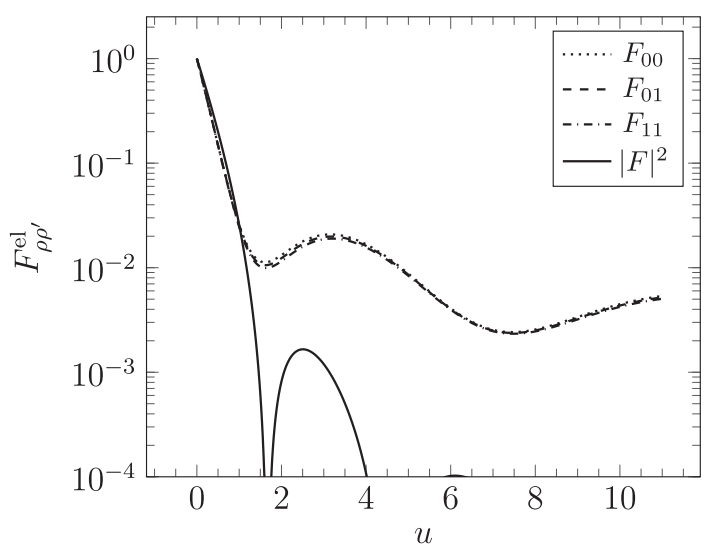

(b)

FIG. 3. Spin structure functions and squared nuclear form factor of (a) ${ }^{83} \mathrm{Kr}$ and (b) ${ }^{125} \mathrm{Te}$ in the elastic channel. 


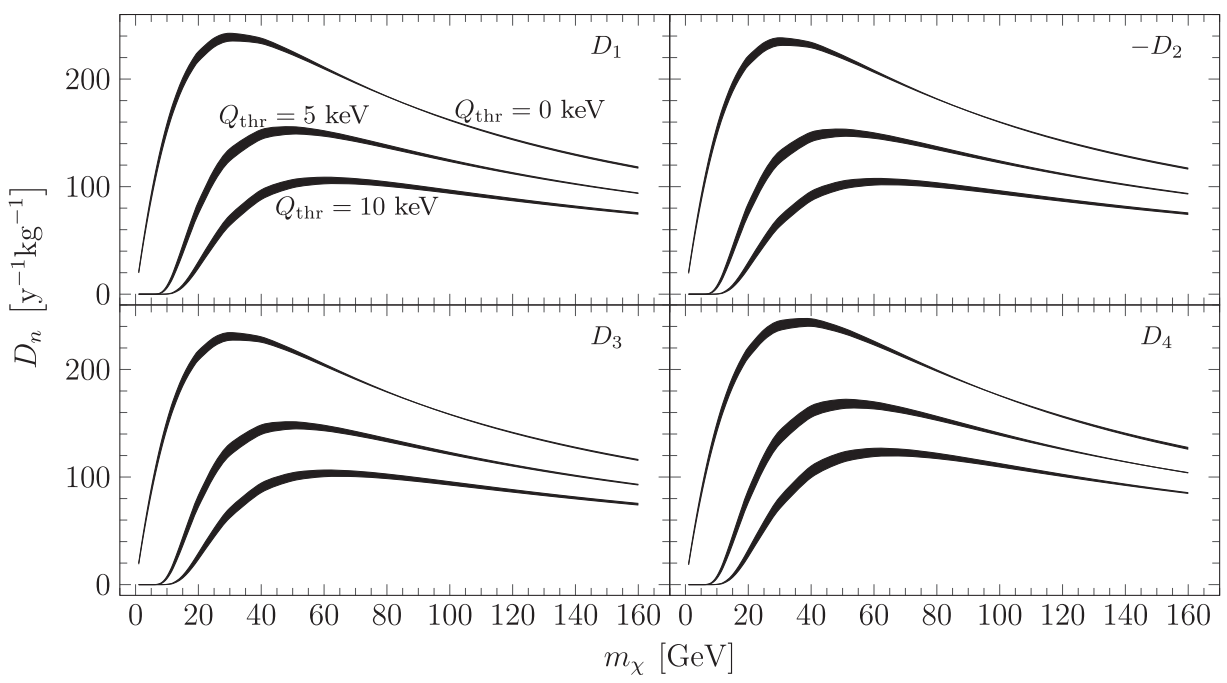

FIG. 4. Nuclear-structure coefficients $D_{n}$ of Eqs. (5)-(8) for elastic scattering off ${ }^{83} \mathrm{Kr}$. The top curves were calculated with $Q_{\text {thr }}=0 \mathrm{keV}$, the middle curves with $Q_{\text {thr }}=5 \mathrm{keV}$, and the bottom curves with $Q_{\mathrm{thr}}=10 \mathrm{keV}$. The thickness of the curves represents the annual modulation. The coefficient $D_{2}$ is given with negative sign to better allow visual comparison.

SSFs drop much faster than for ${ }^{83} \mathrm{Kr}$. For both our target nuclei, the slope of the SSFs briefly turns positive after $u=1$ and the functions remain small but nonzero across the allowed $u$ range. At $u>1$, the values are very small, however, and the main contributions to the event rates come from $0<u<1$. It is also noteworthy that for the elastic channel the three SSFs $F_{\rho \rho^{\prime}}$ are very close to equal.

The nuclear-structure-dependent coefficients $D_{n}$ of Eqs. (5)-(8) were calculated and the values are plotted as a function of the LSP mass in Figs. 4 and 5 for ${ }^{83} \mathrm{Kr}$ and ${ }^{125} \mathrm{Te}$, respectively. Because of the aforementioned sign difference of the isoscalar and isovector SSMEs, the coefficient $D_{2}$ is negative for both ${ }^{83} \mathrm{Kr}$ and ${ }^{125} \mathrm{Te}$. In Figs. 4 and 5 , the values of $-D_{2}$ have thus been plotted to allow visual comparison with the other coefficients. The thickness of the curves represent the annual modulation. The plots were made for three values of the detector threshold energy, $Q_{\mathrm{thr}}=0 \mathrm{keV}$ (top curve), $Q_{\mathrm{thr}}=$ $5 \mathrm{keV}$ (middle curve), and $Q_{\mathrm{thr}}=10 \mathrm{keV}$ (bottom curve). In reality, the threshold is likely somewhere between the two extreme values.

The zero-threshold curves of Figs 4 and 5 peak at roughly $m_{\chi}=30-40 \mathrm{GeV}$ for both ${ }^{83} \mathrm{Kr}$ and ${ }^{125} \mathrm{Te}$. This is the case for both the spin-dependent $\left(D_{1}-D_{3}\right)$ and spinindependent $\left(D_{4}\right)$ channels. Increasing threshold energy moves the peak to somewhat higher masses. For ${ }^{83} \mathrm{Kr}$, the spin-dependent and spin-independent coefficients are quite the same in magnitude, but for ${ }^{125} \mathrm{Te}$ the spin-dependent coefficients are each larger than the spin-independent one by roughly a factor of 2 . In fact, the spin-dependent channel

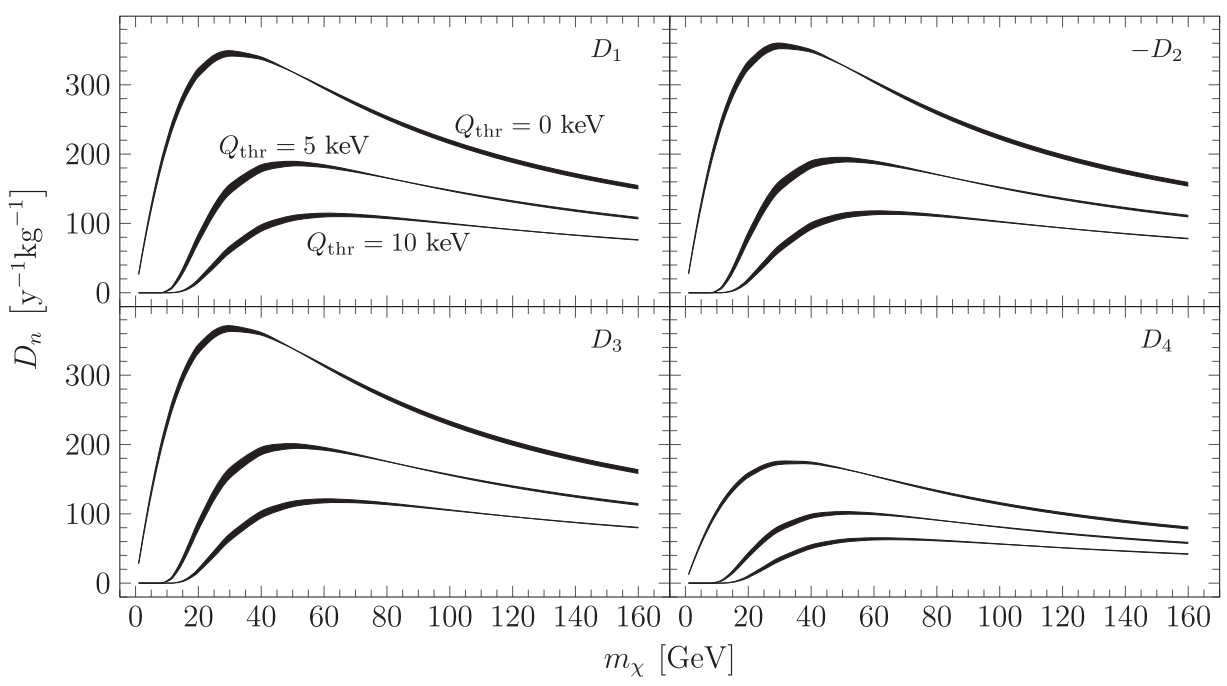

FIG. 5. The same as Fig. 4 for elastic scattering off ${ }^{125} \mathrm{Te}$. 
TABLE VII. Annual averaged $D_{n}$ coefficients of elastic scattering off ${ }^{83} \mathrm{Kr}$ and ${ }^{125} \mathrm{Te}$ with $Q_{\mathrm{thr}}=0$ for select LSP masses. The parameters $a_{n}, b_{n}$, and $c_{n}$ are given to enable the evaluation of the $Q_{\text {thr }}$ dependence of $\bar{D}_{n}$ using Eq. (29). An estimate for any LSP mass between 30 and $200 \mathrm{GeV}$ can be linearly interpolated from the below data.

\begin{tabular}{|c|c|c|c|c|c|c|c|c|c|}
\hline & \multicolumn{6}{|c|}{$m_{\chi}(\mathrm{GeV})$} & \multirow[t]{2}{*}{$a_{n}$} & \multirow[t]{2}{*}{$b_{n}$} & \multirow[t]{2}{*}{$c_{n}$} \\
\hline & 30 & 50 & 80 & 100 & 140 & 200 & & & \\
\hline \multicolumn{10}{|l|}{${ }^{83} \mathrm{Kr}$} \\
\hline $\bar{D}_{1}$ & 238.9 & 224.0 & 183.9 & 161.9 & 129.6 & 99.40 & -0.01944 & -0.62015 & -36.11877 \\
\hline $\bar{D}_{2}$ & -234.8 & -220.6 & -181.5 & -160.0 & -128.4 & -98.73 & -0.01813 & -0.66379 & -35.66811 \\
\hline $\bar{D}_{3}$ & 230.7 & 217.2 & 179.2 & 158.3 & 127.3 & 98.13 & -0.01673 & -0.71160 & -35.16286 \\
\hline $\begin{array}{l}\bar{D}_{4} \\
{ }^{125} \mathrm{Te}\end{array}$ & 240.8 & 236.2 & 199.0 & 175.7 & 140.0 & 106.0 & -0.02898 & 0.33187 & -48.03233 \\
\hline $\bar{D}_{1}$ & 345.3 & 318.5 & 252.3 & 217.7 & 169.3 & 126.5 & -0.04950 & -0.42807 & -58.65738 \\
\hline $\bar{D}_{2}$ & -356.1 & -328.3 & -259.9 & -224.1 & -174.2 & -130.0 & -0.05035 & -0.38671 & -59.19801 \\
\hline $\bar{D}_{3}$ & 367.3 & 338.5 & 267.8 & 230.8 & 179.2 & 133.6 & -0.05115 & -0.34779 & -59.70706 \\
\hline $\bar{D}_{4}$ & 173.6 & 165.3 & 133.0 & 114.9 & 88.91 & 65.68 & -0.05723 & 0.53535 & -71.87464 \\
\hline
\end{tabular}

of ${ }^{125} \mathrm{Te}$ is very strong when compared with the results of Ref. [30], where the same coefficients were calculated for ${ }^{127} \mathrm{I},{ }^{129,131} \mathrm{Xe}$, and ${ }^{133} \mathrm{Cs}$. There ${ }^{129} \mathrm{Xe}$ had the strongest spin-dependent channel, and the coefficients $D_{1}-D_{3}$ peaked at $m_{\chi} \approx 30$ yielding roughly $110 \mathrm{y}^{-1} \mathrm{~kg}^{-1}$ with $Q_{\mathrm{thr}}=0 \mathrm{keV}$. In our calculation for ${ }^{125} \mathrm{Te}$, the same peak lies much higher at $350 \mathrm{y}^{-1} \mathrm{~kg}^{-1}$. The nonzero-threshold graphs are not comparable to the results of Ref. [30] as the integration limits in the present work were corrected to better reflect the exact kinematics of the scattering process. If the spin-independent channel is hindered by some mechanism, as is the case in some supersymmetric models, then ${ }^{125} \mathrm{Te}$ would have an advantage as a detector material.

The annual modulation signal seems to be the most significant for light LSPs, for which $m_{\chi}<80 \mathrm{GeV}$. There is also a mass region visible in each of the graphs of Figs. 4 and 5 where the effect of annual modulation is essentially zero. After this region the modulation amplitude changes sign, which has been predicted to happen at some values of recoil energy or WIMP mass already in some earlier works $[53,54]$. The change of sign was also noted in the calculations of Refs. $[28,30]$ for different detector nuclei. At the favorable low-mass region, the magnitude of the annual modulation can reach roughly $10 \%$ of the total coefficients, but at most WIMP masses the modulation effect is of the order of $1 \%$. With increasing threshold energy, the annual modulation signal becomes slightly larger, as seen in the graphs.

To allow easy extraction of the nuclear-structure coefficients $D_{n}$, we have written

$D_{n}\left(m_{\chi}, Q_{\mathrm{thr}}, \alpha\right)=\bar{D}_{n}\left(m_{\chi}, Q_{\mathrm{thr}}\right)\left[1+M_{n}\left(m_{\chi}, Q_{\mathrm{thr}}\right) \cos \alpha\right]$,

where $\bar{D}_{n}$ is the annual averaged coefficient, $\alpha$ is the phase of the Earth as before, and the annual modulation amplitude is contained in the $M_{n}$ term. The $Q_{\text {thr }}$ dependence of the annual averaged $D_{n}$ coefficients can be fitted by the exponential

$$
\bar{D}_{n}\left(m_{\chi}, Q_{\mathrm{thr}}\right)=\bar{D}\left(m_{\chi}, 0\right) e^{\left(a_{n}+b_{n} / \mu_{r}+c_{n} / \mu_{r}^{2}\right) Q_{\mathrm{thr}}},
$$

where the parameters $a_{n}, b_{n}$, and $c_{n}$ as well as the zerothreshold values of the $\bar{D}_{n}$ coefficients for a range of LSP masses are given in Table VII. To access the annual modulation, we write the $M_{n}$ coefficients as

$$
M_{n}\left(m_{\chi}, Q_{\mathrm{thr}}\right)=M_{n}\left(m_{\chi}, 0\right)+\left(\beta_{n}+\gamma_{n} / \mu_{r}+\delta_{n} / \mu_{r}^{2}\right) Q_{\mathrm{thr}} .
$$

The zero-threshold values $M_{n}\left(m_{\chi}, 0\right)$ and the parameters $\beta_{n}, \gamma_{n}$, and $\delta_{n}$ are given in Table VIII. One can linearly interpolate the data of Tables VII and VIII to find a reasonable estimate of the zero-threshold coefficients for any given LSP mass.

The fit described by Eqs. (28)-(30) is by no means exact, but provides an easy-to-compute way to reach a good estimate of the coefficients $D_{n}$ and annual modulation. The accuracy of the fit is demonstrated in Table IX. Small modeling errors in the fit parameters as well as the approximation of linear interpolation of the zero-threshold coefficients for other LSP masses than those in Tables VII and VIII will cause discrepancies between the exact computed results and the fitted ones. From Table IX, however, we find that these discrepancies are relatively small.

As a final remark, we briefly compare the annual averaged $D_{n}$ coefficients for the full and truncated jj44b calculations of the ${ }^{83} \mathrm{Kr}$ case as well as the truncations 1 and 2 of the ${ }^{125} \mathrm{Te}$ case, which were described in Sec. II. These were considered the most reasonable of the performed truncations when comparing the computed and experimental electromagnetic properties. In the ${ }^{125} \mathrm{Te}$ case, going 
TABLE VIII. Zero-threshold annual modulation amplitudes $M_{n}\left(m_{\chi}, Q_{\mathrm{thr}}=0\right)$ of elastic scattering off ${ }^{83} \mathrm{Kr}$ and ${ }^{125} \mathrm{Te}$ for select LSP masses. The parameters $\beta_{n}, \gamma_{n}$, and $\delta_{n}$ are given for calculating the $Q_{\text {thr }}$ dependence of modulation from Eq. (30). An estimate for any LSP mass between 30 and $200 \mathrm{GeV}$ can be linearly interpolated from the below data.

\begin{tabular}{|c|c|c|c|c|c|c|c|c|c|}
\hline & \multicolumn{6}{|c|}{$m_{\chi}(\mathrm{GeV})$} & \multirow[t]{2}{*}{$\beta_{n}$} & \multirow[t]{2}{*}{$\gamma_{n}$} & \multirow[t]{2}{*}{$\delta_{n}$} \\
\hline & 30 & 50 & 80 & 100 & 140 & 200 & & & \\
\hline \multicolumn{10}{|l|}{${ }^{83} \mathrm{Kr}$} \\
\hline$M_{1}$ & 0.0152 & 0.0070 & 0.0005 & -0.0017 & -0.0040 & -0.0056 & 0.00045 & 0.01188 & 1.85911 \\
\hline$M_{2}$ & 0.0153 & 0.0072 & 0.0009 & -0.0013 & -0.0035 & -0.0050 & 0.00046 & 0.01189 & 1.85491 \\
\hline$M_{3}$ & 0.0154 & 0.0074 & 0.0012 & -0.0008 & -0.0029 & -0.0043 & 0.00047 & 0.01192 & 1.85004 \\
\hline $\begin{array}{l}M_{4} \\
{ }^{125} \mathrm{Te}\end{array}$ & 0.0185 & 0.0108 & 0.0029 & -0.0005 & -0.0050 & -0.0088 & -0.00037 & 0.04569 & 1.47578 \\
\hline$M_{1}$ & 0.0114 & 0.0003 & -0.0089 & -0.0121 & -0.0155 & -0.0177 & 0.00045 & 0.01725 & 2.76392 \\
\hline$M_{2}$ & 0.0114 & 0.0002 & -0.0090 & -0.0122 & -0.0157 & -0.0179 & 0.00042 & 0.01870 & 2.74522 \\
\hline$M_{3}$ & 0.0114 & 0.0002 & -0.0091 & -0.0124 & -0.0159 & -0.0181 & 0.00040 & 0.02006 & 2.72755 \\
\hline$M_{4}$ & 0.0137 & 0.0026 & -0.0077 & -0.0117 & -0.0165 & -0.0201 & -0.00044 & 0.06341 & 2.11983 \\
\hline
\end{tabular}

from truncation 1 to truncation 2 leads to very small changes in the $\bar{D}_{n}$ coefficients for $n=1,2,3$, no more than $2 \%$ at any given LSP mass. Going from full to truncated calculation in the ${ }^{83} \mathrm{Kr}$ case leads to maximum change of $7 \%$ in the $\bar{D}_{3}, 4 \%$ in the $\bar{D}_{1}$, and $2 \%$ in the $\bar{D}_{2}$ coefficient. An exception is the $D_{4}$ coefficient, which remains practically unchanged $(<0.5 \%)$ in the truncation for both nuclei. The elastic channel, especially the spinindependent part, is thus quite stable with respect to reasonably small changes in nuclear structure.

\section{B. Results for inelastic scattering}

The SSMEs related to inelastic LSP-nucleus scattering are presented in Table $\mathrm{X}$ for each of the truncation schemes presented in Sec. II. We find that the values of the SSMEs generally follow the trend of the $B(\mathrm{M} 1)$ values of Table IV for each truncation. The JUN45 calculation for ${ }^{83} \mathrm{Kr}$ produces SSMEs of the same sign, but they are likely to be underestimated because of the nearly vanishing $B(\mathrm{M} 1)$ value we computed with this interaction. We thus expect the full jj44b calculation for ${ }^{83} \mathrm{Kr}$ and truncation 1 for ${ }^{125} \mathrm{Te}$ to yield the most reliable results for the SSMEs and structure factors, and we employ only those schemes in the following calculations and discussion.

The values of the SSMEs of ${ }^{83} \mathrm{Kr}$ are very small and inevitably lead to an inelastic scattering event rate which cannot compete with the well-established detector nuclei. For ${ }^{125} \mathrm{Te}$, the SSMEs are larger by a factor of 3 and should

TABLE IX. Comparison between calculated $\bar{D}_{n}$ and $M_{n}$ coefficients and those fitted by Eqs. (28)-(30). The comparison is made for $\bar{D}_{1}$ (columns 2-3) and $M_{1}$ (columns 6-7) with $m_{\chi}=50 \mathrm{GeV}$ and for $\bar{D}_{4}$ (columns 4-5) and $M_{4}$ (columns 8-9) with $m_{\chi}=126 \mathrm{GeV}$. In the "fit" case, the zero-threshold values of $\bar{D}_{4}$ and $M_{4}$ have been linearly interpolated from the data of Tables VII and VIII.

\begin{tabular}{|c|c|c|c|c|c|c|c|c|}
\hline \multirow[b]{2}{*}{$Q_{\mathrm{thr}}(\mathrm{keV})$} & \multicolumn{2}{|c|}{$\begin{array}{l}\bar{D}_{1}\left(\mathrm{~kg}^{-1} \mathrm{y}^{-1}\right) \\
m_{\chi}=50 \mathrm{GeV}\end{array}$} & \multicolumn{2}{|c|}{$\begin{array}{c}\bar{D}_{4}\left(\mathrm{~kg}^{-1} \mathrm{y}^{-1}\right) \\
m_{\chi}=126 \mathrm{GeV}\end{array}$} & \multicolumn{2}{|c|}{$\begin{array}{c}\quad M_{1} \\
m_{\chi}=50 \mathrm{GeV} \\
\end{array}$} & \multicolumn{2}{|c|}{$\begin{array}{c}M_{4} \\
m_{\chi}=126 \mathrm{GeV}\end{array}$} \\
\hline & Fit & Calc. & Fit & Calc. & Fit & Calc. & Fit & Calc. \\
\hline \multicolumn{9}{|l|}{${ }^{83} \mathrm{Kr}$} \\
\hline 0 & 224.0 & 224.0 & 152.5 & 151.0 & 0.0070 & 0.0070 & -0.0034 & -0.0037 \\
\hline 5 & 151.1 & 152.3 & 123.1 & 122.3 & 0.0212 & 0.0223 & 0.0027 & 0.0026 \\
\hline 10 & 101.9 & 102.7 & 99.39 & 98.84 & 0.0355 & 0.0368 & 0.0088 & 0.0087 \\
\hline 15 & 68.72 & 68.95 & 80.24 & 79.62 & 0.0497 & 0.0507 & 0.0149 & 0.0147 \\
\hline 20 & 46.35 & 46.09 & 64.77 & 63.95 & 0.0639 & 0.0640 & 0.0209 & 0.0205 \\
\hline 25 & 31.26 & 30.75 & 52.28 & 51.22 & 0.0781 & 0.0769 & 0.0270 & 0.0262 \\
\hline \multicolumn{9}{|l|}{${ }^{125} \mathrm{Te}$} \\
\hline 0 & 318.5 & 318.5 & 98.01 & 96.70 & 0.0003 & 0.0003 & -0.0148 & -0.0152 \\
\hline 5 & 184.3 & 186.2 & 69.79 & 69.48 & 0.0163 & 0.0179 & -0.0089 & -0.0090 \\
\hline 10 & 106.6 & 107.6 & 49.70 & 49.52 & 0.0322 & 0.0344 & -0.0030 & -0.0031 \\
\hline 15 & 61.67 & 61.62 & 35.39 & 34.98 & 0.0482 & 0.0501 & 0.0029 & 0.0027 \\
\hline 20 & 35.68 & 35.02 & 25.20 & 24.68 & 0.0641 & 0.0650 & 0.0088 & 0.0084 \\
\hline 25 & 20.64 & 19.81 & 17.95 & 17.18 & 0.0801 & 0.0794 & 0.0146 & 0.0139 \\
\hline
\end{tabular}


TABLE X. Static spin matrix elements in case of inelastic LSPnucleus scattering off ${ }^{83} \mathrm{Kr}$ and ${ }^{125} \mathrm{Te}$.

\begin{tabular}{lccc}
\hline \hline Nucleus & Setup & $\Omega_{0}^{\text {in }}$ & $\Omega_{1}^{\text {in }}$ \\
\hline${ }^{83} \mathrm{Kr}$ & Full jj44b & -0.048 & 0.044 \\
& Truncated jj44b & -0.053 & 0.027 \\
& Full JUN45 & 0.004 & 0.003 \\
${ }^{125} \mathrm{Te}$ & Truncation 1 & -0.157 & 0.196 \\
& Truncation 2 & -0.162 & 0.205 \\
& Truncation 3 & -0.0284 & 0.0463 \\
& Truncation 4 & -0.0055 & 0.0005 \\
\hline \hline
\end{tabular}

not hinder the event rates too much. The signs of the isovector and isoscalar matrix elements are again opposite, as in the elastic case.

The spin structure functions in the inelastic case are presented in Fig 6 for (a) ${ }^{83} \mathrm{Kr}$ and (b) ${ }^{125} \mathrm{Te}$. Here, contrary to the elastic case, the structure functions deviate from each other substantially in the $0<u<2$ region, especially for ${ }^{125} \mathrm{Te}$. For larger $u$, the structure functions become

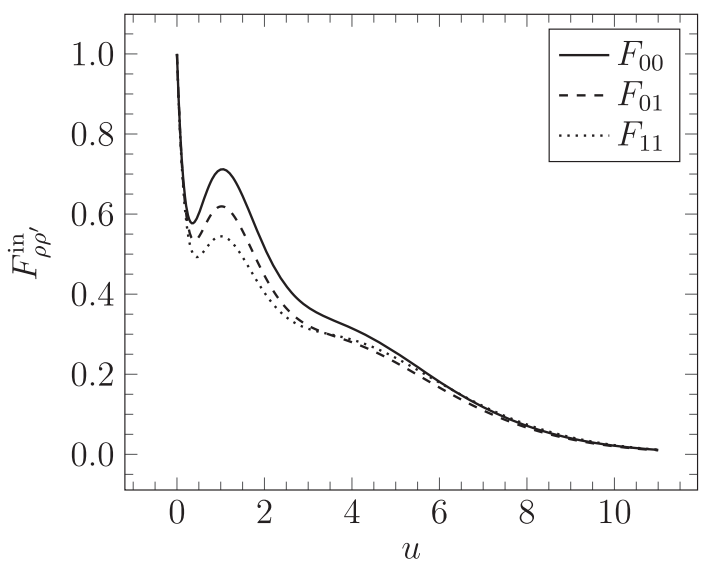

(a)

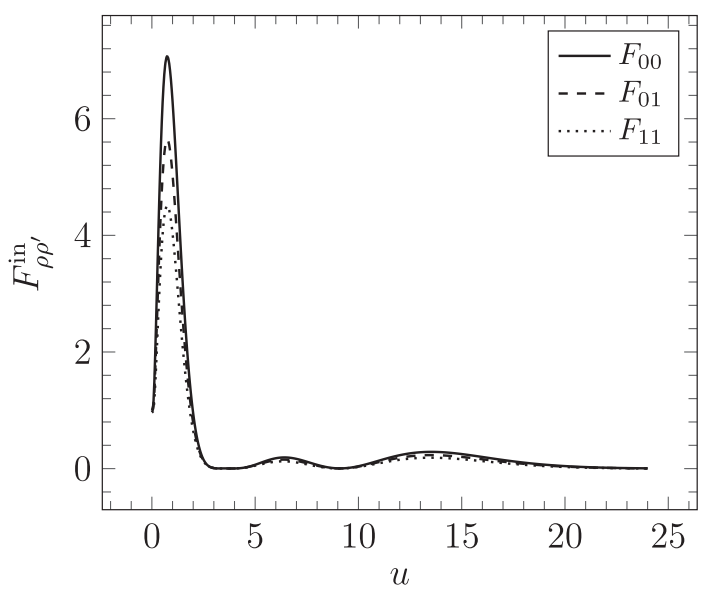

(b)

FIG. 6. Spin structure functions of (a) ${ }^{83} \mathrm{Kr}$ and (b) ${ }^{125} \mathrm{Te}$ in the inelastic channel. approximately equal again. The SSFs of ${ }^{83} \mathrm{Kr}$ begin with a steep downward slope and stay below unity for all $u$. For ${ }^{125} \mathrm{Te}$, the situation is reversed, and the SSFs begin with an upward slope reaching a peak at $u \approx 0.8$, then vanish very quickly and become essentially zero at $u \approx 3$. There are a few small bumps for $u>3$, which can contribute to the event rate for a sufficiently heavy LSP. The major contribution, however, still comes from $0<u<3$. For ${ }^{83} \mathrm{Kr}$, the SSFs tend to zero much slower than for ${ }^{125} \mathrm{Te}$, although this is not likely to make up for the small SSME.

The SSFs can be compared to those calculated in Ref. [33] for ${ }^{129} \mathrm{Xe}$ and ${ }^{131} \mathrm{Xe}$. Our results convert into the form used in Ref. [33] as

$$
\begin{gathered}
S_{00}(u)=\frac{2 J_{\mathrm{i}}+1}{16 \pi} \Omega_{0}^{2} F_{00}(u), \\
S_{01}(u)=\frac{2 J_{\mathrm{i}}+1}{8 \pi} \Omega_{0} \Omega_{1} F_{01}(u), \\
S_{11}(u)=\frac{2 J_{\mathrm{i}}+1}{16 \pi} \Omega_{1}^{2} F_{11}(u) .
\end{gathered}
$$

We find that our inelastic structure functions for ${ }^{83} \mathrm{Kr}$ are much smaller overall than the form factors obtained for the

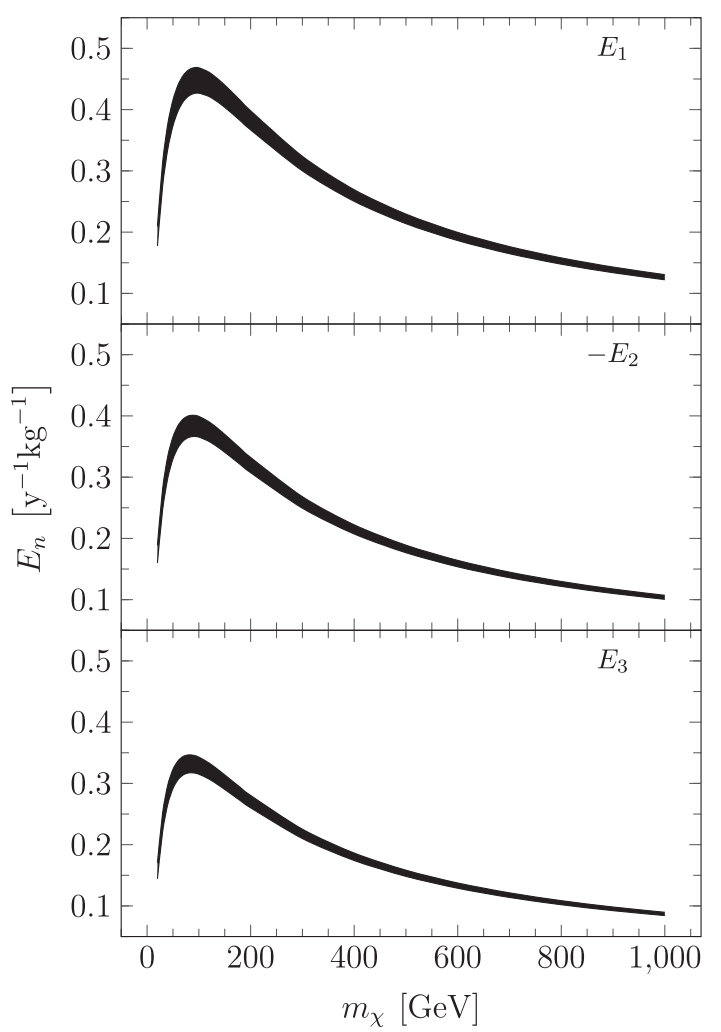

FIG. 7. Nuclear-structure coefficients $E_{n}$ of Eqs. (21)-(23) for inelastic scattering off ${ }^{83} \mathrm{Kr}$. The thickness of the curves represents the annual modulation. The coefficient $E_{2}$ is given with negative sign to better allow visual comparison. 
xenons in Ref. [33]. The SSFs of ${ }^{125} \mathrm{Te}$ have a similar shape in the important $0<u<3$ region to the SSFs of Ref. [33], but are larger than the upper limit of the ${ }^{129} \mathrm{Xe} S$ functions by roughly a factor of 2 for $S_{00}, 3$ for $S_{01}$, and a factor of 4 for $S_{11}$. Our ${ }^{125} \mathrm{Te}$ SSFs are also larger than the upper limits of ${ }^{131} \mathrm{Xe} S$ functions by $10 \%$ for $S_{00}$ and $S_{01}$, and $90 \%$ for $S_{11}$. Compared to ${ }^{125} \mathrm{Te},{ }^{131} \mathrm{Xe}$ suffers also from the kinematical disadvantage of a higher $80 \mathrm{keV}$ first excited state. The shell-model calculation used to describe ${ }^{129,131} \mathrm{Xe}$ in Refs. [32,33] reproduced the experimental energy spectra remarkably well and the resulting magnetic moments were in slightly better agreement with experiment than those computed in the present work. Thus a larger modeling error is expected in our calculation, and making conclusions by directly comparing these two results is difficult.

We plot the nuclear structure coefficients $E_{n}$ of Eqs. (21)-(23) as a function of the LSP mass in Figs. 7 and 8 for ${ }^{83} \mathrm{Kr}$ and ${ }^{125} \mathrm{Te}$, respectively. The plots are made for a wider mass range than in the elastic case to accommodate the full qualitative behavior of the coefficients. For ${ }^{83} \mathrm{Kr}$, the values of each $E_{n}$ are quite small as predicted by the small SSMEs. The curves peak at $m_{\chi} \approx 100 \mathrm{GeV}$. For ${ }^{125} \mathrm{Te}$, the values of the $E$ coefficients are much more favorable. Peaking at $m_{\chi} \approx 150 \mathrm{GeV}$, the curves reach a maximum of roughly $16 \mathrm{y}^{-1} \mathrm{~kg}^{-1}$. Provided that the LSP is sufficiently heavy, the nuclear response of ${ }^{125} \mathrm{Te}$ appears very promising.

In inelastic scattering, the annual modulation signal is percentually more visible than in the case of elastic scattering. We see in Figs. 7 and 8 that the magnitude of the modulation signal is at best more than $10 \%$ of the coefficients $E_{n}$. It is noteworthy, that contrary to elastic scattering, here the modulation signal does not change sign for any $m_{\chi}<1000 \mathrm{GeV}$. The event rate, as well as the modulation signal, for ${ }^{83} \mathrm{Kr}$ is likely to be too low to be measured with present equipment, but inelastic

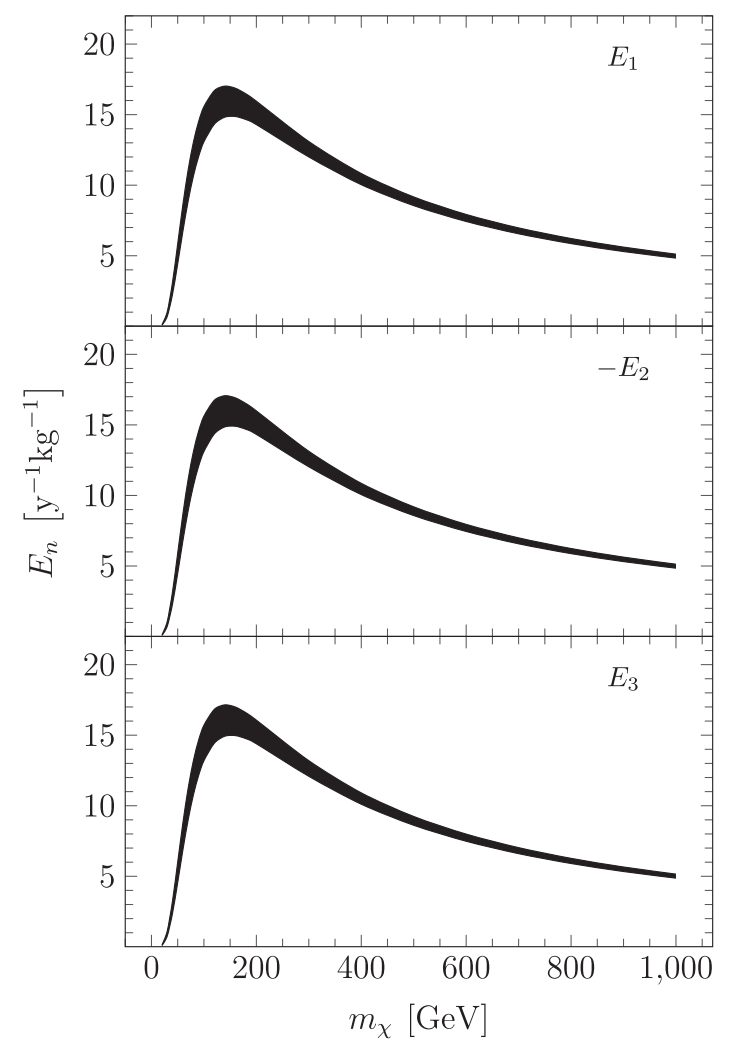

FIG. 8. The same as Fig. 7 for inelastic scattering off ${ }^{125}$ Te.

LSP-nucleus scattering off ${ }^{125} \mathrm{Te}$ might offer new possibilites for dark-matter detection.

Analogously to the elastic channel, we can provide a formula for easy extraction of the $E_{n}$ coefficients. In the inelastic case, however, there is no $Q_{\text {thr }}$ dependence. The annual average values $\bar{E}_{n}$ for both ${ }^{83} \mathrm{Kr}$ and ${ }^{125} \mathrm{Te}$ are given in Table XI and the modulation amplitudes $M_{n}$ in Table XII. The results can again be linearly interpolated for other LSP masses within reasonable accuracy. Using the data of tables XI and XII, one can find the $E_{n}$ coefficients for a given LSP mass as

TABLE XI. Annual averaged $E_{n}$ coefficients of inelastic scattering off ${ }^{83} \mathrm{Kr}$ and ${ }^{125} \mathrm{Te}$ for select LSP masses. An estimate for any LSP mass between 30 and $1000 \mathrm{GeV}$ can be linearly interpolated from the below data.

\begin{tabular}{|c|c|c|c|c|c|c|c|c|c|}
\hline & \multicolumn{9}{|c|}{$m_{\chi}(\mathrm{GeV})$} \\
\hline & 30 & 50 & 80 & 100 & 140 & 200 & 300 & 500 & 1000 \\
\hline \multicolumn{10}{|l|}{${ }^{83} \mathrm{Kr}$} \\
\hline $\bar{E}_{1}$ & 0.294 & 0.395 & 0.443 & 0.448 & 0.429 & 0.383 & 0.312 & 0.222 & 0.126 \\
\hline $\bar{E}_{2}$ & -0.264 & -0.348 & -0.382 & -0.382 & -0.362 & -0.320 & -0.259 & -0.183 & -0.104 \\
\hline $\begin{array}{l}\bar{E}_{3} \\
125 \mathrm{Te}\end{array}$ & 0.236 & 0.307 & 0.332 & 0.329 & 0.308 & 0.270 & 0.218 & 0.153 & 0.087 \\
\hline $\bar{E}_{1}$ & 0.853 & 5.128 & 11.850 & 14.319 & 15.921 & 15.121 & 12.546 & 8.859 & 4.971 \\
\hline $\bar{E}_{2}$ & -0.887 & -5.189 & -11.902 & -14.366 & -15.963 & -15.161 & -12.581 & -8.885 & -4.986 \\
\hline $\bar{E}_{3}$ & 0.931 & 5.274 & 11.987 & 14.447 & 16.038 & 15.229 & 12.639 & 8.927 & 5.011 \\
\hline
\end{tabular}


TABLE XII. Annual modulation amplitudes $M_{n}\left(m_{\chi}\right)$ of inelastic scattering off ${ }^{83} \mathrm{Kr}$ and ${ }^{125}$ Te for select LSP masses. An estimate for any LSP mass between 30 and $1000 \mathrm{GeV}$ can be linearly interpolated from the below data.

\begin{tabular}{lccccccccc}
\hline \hline & \multicolumn{7}{c}{$m_{\chi}(\mathrm{GeV})$} \\
\cline { 2 - 10 } & 30 & 50 & 80 & 100 & 140 & 200 & 300 & 500 & 1000 \\
\hline${ }^{83} \mathrm{Kr}$ & & & & & & & & \\
$M_{1}$ & 0.06604 & 0.05343 & 0.04815 & 0.04634 & 0.04365 & 0.04088 & 0.03809 & 0.03541 & 0.03311 \\
$M_{2}$ & 0.06547 & 0.05205 & 0.04617 & 0.04424 & 0.04156 & 0.03891 & 0.03630 & 0.03380 & 0.03166 \\
$M_{3}$ & 0.06497 & 0.05085 & 0.04443 & 0.04242 & 0.03982 & 0.03740 & 0.03511 & 0.03297 & 0.03114 \\
${ }_{125} \mathrm{Te}$ & & & & & & & & & \\
$M_{1}$ & 0.18836 & 0.13859 & 0.10163 & 0.08708 & 0.06909 & 0.05485 & 0.04363 & 0.03476 & 0.02832 \\
$M_{2}$ & 0.18723 & 0.13818 & 0.10154 & 0.08707 & 0.06915 & 0.05494 & 0.04375 & 0.03488 & 0.02843 \\
$M_{3}$ & 0.18598 & 0.13768 & 0.10141 & 0.08704 & 0.06921 & 0.05505 & 0.04388 & 0.03502 & 0.02858 \\
\hline \hline
\end{tabular}

$$
E_{n}\left(m_{\chi}, \alpha\right)=\bar{E}_{n}\left(m_{\chi}\right)\left[1+M_{n}\left(m_{\chi}\right) \cos \alpha\right]
$$

When making a comparison of annual averaged $E_{n}$ coefficients between the full and truncated jj44b calculations of the ${ }^{83} \mathrm{Kr}$ case as well as the truncations 1 and 2 of the ${ }^{125} \mathrm{Te}$ case we find that there is a larger change with respect to truncation than in the elastic case. For ${ }^{125} \mathrm{Te}$, the $\bar{E}_{n}$ coefficients change a maximum of $7 \%$ at any given LSP mass when going from truncation 1 to truncation 2. For ${ }^{83} \mathrm{Kr}$, there is a maximum change of $17 \%$ in $\bar{E}_{1}, 34 \%$ in $\bar{E}_{2}$, and $62 \%$ in $\bar{E}_{3}$ when going from the full to the truncated calculation. This is mostly due to the isovector SSME being significantly smaller in the truncated calculation while the isoscalar SSME remained quite unchanged. The inelastic channel is rather sensitive to change in nuclear structure, and the event rate, with respect to truncation, behaves in a trend similar to the computed $B(\mathrm{M} 1)$ value for the corresponding transition.

\section{CONCLUSIONS}

In this work, we have examined event rates of elastic and inelastic scattering of an LSP off ${ }^{83} \mathrm{Kr}$ and ${ }^{125} \mathrm{Te}$. The wave functions of the relevant states of the target nuclei were computed in the framework of the nuclear shell model using state-of-the-art two-body interactions. The nuclearstructure contribution to the event rate was separated and evaluated independently of any supersymmetric model.

Comparing the nuclear-structure coefficients of Figs. 4,5 (elastic scattering) and 7,8 (inelastic scattering), it is evident that the nuclear response of ${ }^{125} \mathrm{Te}$ is more favorable to LSP scattering. While the elastic channel is quite strong for both nuclei, the small SSMEs of ${ }^{83} \mathrm{Kr}$ make the inelastic channel very weak. In ${ }^{125} \mathrm{Te}$, the nuclear response to inelastic scattering appears much more favorable.

By using the nuclear-structure-dependent coefficients, one can easily insert a choice of supersymmetric parameters to calculate the total event rate of LSP-nucleus scattering. Some such SUSY solutions are given in Ref. [24]. The hierarchy between ${ }^{83} \mathrm{Kr}$ and ${ }^{125} \mathrm{Te}$ predicted by the nuclear structure parts is visible in calculated total event rates. Although the coherent nuclear structure coefficient $D_{4}$ of ${ }^{83} \mathrm{Kr}$ is larger than that of ${ }^{125} \mathrm{Te}$, the $A^{2}$ dependence gives ${ }^{125} \mathrm{Te}$ an advantage also in SUSY models which favor the coherent channel. A large portion of the LSP parameter space is already excluded by dark matter search at the LHC or other direct detection experiments. Many of the SUSY solutions of Ref. [24] are thus probably obsolete.

Even though appreciable theoretical event rates can be obtained for ${ }^{125} \mathrm{Te}$, there is at present no guarantee of experimental feasibility. The natural abundance of ${ }^{125} \mathrm{Te}$ is quite low, and there could be other factors which make it an unreasonable detector material, such as quenching factors. Nevertheless, the theoretical calculations presented here give an incentive for a study on the experimental aspects of a WIMP detector using ${ }^{125} \mathrm{Te}$ as a detector material. Initial consideration of experimental aspects has been made recently in Ref. [37].

\section{ACKNOWLEDGMENTS}

This work has been partially supported by the Academy of Finland under the Finnish Centre of Excellence Programme 2012-2017 (Nuclear and Accelerator Based Programme at JYFL) and the FIDIPRO program. P. Pirinen was supported by a graduate student stipend from the Magnus Ehrnrooth Foundation. 
[1] F. Zwicky, Helv. Phys. Acta 6, 110 (1933).

[2] E. Komatsu et al., Astrophys. J. Suppl. Ser. 192, 18 (2011).

[3] The Planck Collaboration, P. A. R. Ade et al., Astron. Astrophys. 571, A16 (2014).

[4] C. Alcock et al., Astrophys. J. 542, 281 (2000).

[5] P. Tisserand et al., Astron. Astrophys. 469, 387 (2007).

[6] H. C. Cheng, J. L. Feng, and K. T. Matchev, Phys. Rev. Lett. 89, 211301 (2002).

[7] V. K. Oikonomou, J. D. Vergados, and C. C. Moustakidis, Nucl. Phys. B773, 19 (2007).

[8] S. Nussinov, Phys. Lett. B 165, 55 (1985).

[9] S. B. Gudnason, C. Kouvaris, and F. Sannino, Phys. Rev. D 74, 095008 (2006).

[10] J. Hubisz and P. Meade, Phys. Rev. D 71, 035016 (2005).

[11] A. Birkedal, A. Noble, M. Perelstein, and A. Spray, Phys. Rev. D 74, 035002 (2006).

[12] G. Jungman, M. Kamionkowski, and K. Griest, Phys. Rep. 267, 195 (1996).

[13] J. Ellis and J. S. Hagelin, Nucl. Phys. B238, 453 (1984).

[14] T. A. Girard et al., Phys. Lett. B 621, 233 (2005).

[15] M. Barnabé-Heider et al., Phys. Lett. B 624, 186 (2005).

[16] D. S. Akerib et al., Phys. Rev. D 72, 052009 (2005).

[17] A. Broniatowski et al., Phys. Lett. B 681, 305 (2009).

[18] H. S. Lee et al., Phys. Rev. D 90, 052006 (2014).

[19] R. Bernabei et al., Nucl. Instrum. Methods Phys. Res., Sect. A 592, 297 (2008).

[20] E. Aprile et al., Phys. Rev. Lett. 105, 131302 (2010).

[21] K. Abe et al., Nucl. Instrum. Methods Phys. Res., Sect. A 716, 78 (2013).

[22] V. A. Kudryavtsev (for the LZ Collaboration), AIP Conf. Proc. 1672, 060003 (2015).

[23] J. Ellis, R. A. Flores, and J. D. Lewin, Phys. Lett. B 212, 375 (1988).

[24] J. D. Vergados and T. S. Kosmas, Phys. At. Nucl. 61, 1066 (1998).

[25] J. Ellis and R. A. Flores, Nucl. Phys. B307, 883 (1988).

[26] J. Engel and P. Vogel, Phys. Rev. D 40, 3132 (1989).

[27] F. Iachello and L. M. Krauss, Phys. Lett. B 254, 220 (1991).

[28] M. Kortelainen, T. S. Kosmas, J. Suhonen, and J. Toivanen, Phys. Lett. B 632, 226 (2006).

[29] E. Holmlund, M. Kortelainen, T. S. Kosmas, J. Suhonen, and J. Toivanen, Phys. Lett. B 584, 31 (2004).

[30] P. Toivanen, M. Kortelainen, J. Suhonen, and J. Toivanen, Phys. Rev. C 79, 044302 (2009).

[31] M. T. Ressell and D. J. Dean, Phys. Rev. C 56, 535 (1997).
[32] J. Menéndez, D. Gazit, and A. Schwenk, Phys. Rev. D 86, 103511 (2012).

[33] L. Baudis, G. Kessler, P. Klos, R. F. Lang, J. Menéndez, S. Reichard, and A. Schwenk, Phys. Rev. D 88, 115014 (2013).

[34] L. Vietze, P. Klos, J. Menéndez, W. C. Haxton, and A. Schwenk, Phys. Rev. D 91, 043520 (2015).

[35] P. Klos, J. Menéndez, D. Gazit, and A. Schwenk, Phys. Rev. D 88, 083516 (2013).

[36] J. D. Vergados, F. T. Avignone III, P. Pirinen, P. C. Srivastava, M. Kortelainen, and J. Suhonen, Phys. Rev. D 92, 015015 (2015).

[37] J. D. Vergados, F. T. Avignone III, M. Kortelainen, P. Pirinen, P. C. Srivastava, J. Suhonen, and A. W. Thomas, arXiv:1601.06813.

[38] A. K. Drukier, K. Freese, and D. N. Spergel, Phys. Rev. D 33, 3495 (1986).

[39] R. Bernabei et al., Eur. Phys. J. C 73, 2648 (2013).

[40] C. E. Aalseth et al., Phys. Rev. Lett. 107, 141301 (2011).

[41] K. Freese, M. Lisanti, and C. Savage, Rev. Mod. Phys. 85, 1561 (2013).

[42] B. Cheal et al., Phys. Rev. Lett. 104, 252502 (2010); see also B. A. Brown and A. F. Lisetskiy (unpublished).

[43] B. A. Brown, W. D. M. Rae, E. McDonald, and M. Horoi, NuShellX@MSU, http://www.nscl.msu.edu/ $\sim$ brown/resources/resources.html.

[44] R. Machleidt, F. Sammarruca, and Y. Song, Phys. Rev. C 53, R1483 (1996).

[45] B. A. Brown, N. J. Stone, J. R. Stone, I. S. Towner, and M. Hjorth-Jensen, Phys. Rev. C 71, 044317 (2005).

[46] National Nuclear Data Center: Evaluated Nuclear Structure Data File, http://www.nndc.bnl.gov/ensdf/.

[47] M. Honma, T. Otsuka, T. Mizusaki, and M. Hjorth-Jensen, Phys. Rev. C 80, 064323 (2009).

[48] S. K. Chamoli, A. E. Stuchbery, and M. C. East, Phys. Rev. C 80, 054301 (2009).

[49] F. J. Kerr and D. Lynden-Bell, Mon. Not. R. Astron. Soc. 221, 1023 (1986).

[50] P. J. McMillan and J. J. Binney, Mon. Not. R. Astron. Soc. 402, 934 (2010).

[51] M. J. Reid et al., Astrophys. J. 700, 137 (2009).

[52] T. Piffl et al., Astron. Astrophys. 562, A91 (2014).

[53] J. D. Lewin and P. F. Smith, Astropart. Phys. 6, 87 (1996).

[54] M. J. Lewis and K. Freese, Phys. Rev. D 70, 043501 (2004). 